\title{
Association of the Catechol O-Methyltransferase Val158-Met Polymorphism and Reduced Interference Control in Korean Children with Attention-Deficit Hyperactivity Disorder
}

\author{
Subin Park', Jong-Eun Park², Hee Jeong Yoo ${ }^{2}$, Jae-Won Kim ${ }^{3}$ Jae Hoon Cheong ${ }^{4}$, \\ Doug Hyun $\mathrm{Han}^{5}$, Yeni Kim${ }^{1}$, and Bung-Nyun $\mathrm{Kim}^{3 凶}$ \\ ${ }^{1}$ Department of Psychiatry, Seoul National Hospital, Seoul, Republic of Korea \\ 2Department of Neuropsychiatry, Seoul National University Bundang Hospital, Seongnam, Republic of Korea \\ ${ }^{3}$ Division of Child and Adolescent Psychiatry, Department of Psychiatry, Seoul National University Hospital, Seoul, Republic of Korea \\ ${ }^{4}$ Uimyung Research Institute for Neuroscience, Sahmyook University, Seoul, Republic of Korea \\ ${ }^{5}$ Department of Psychiatry, Chung-Ang University College of Medicine, Seoul, Republic of Korea
}

Objective We tested for association of the catechol-O-methyltransferase (COMT) Val ${ }^{158}$-Met (rs4680) polymorphism with attentiondeficit hyperactivity disorder (ADHD) using family-based test in Korean trios.

Methods A total of 181 subjects with ADHD along with both of their biological parents were recruited from University Hospitals in Korea. We performed a transmission disequilibrium test (TDT) on 181 trios.

Results In the TDT, we found the over-transmission of the Val allele in children with $\operatorname{ADHD}\left(\chi^{2}=4.21, \mathrm{p}=0.040\right)$.

Conclusion These results suggest that the COMT Val158-Met polymorphism is associated with ADHD among the Korean population. However, this study must be replicated in larger populations.

Psychiatry Investig 2015;12(4):563-565

Key Words Attention-deficit hyperactivity disorder, COMT.

\section{INTRODUCTION}

Attention-deficit hyperactivity disorder (ADHD) is a common disorder that has about $4-10 \%$ prevalence rate among elementary school children 1 and has an estimated heritability of approximately $80 \% .^{2}$ Dysregulation of the central dopaminergic and noradrenergic systems is known to be involved in pathophysiology of ADHD. ${ }^{3}$ Catechol-O-methyltransferase (COMT) degrades synaptic dopamine and noradrenaline and plays a specific role in the catabolism of prefrontal cortical dopamine. ${ }^{4}$ The COMT gene has a functional polymorphism at codon 158 that results in a single amino acid change [methionine (Met) for valine (Val)]. The enzyme activities are high-

Received: September 27, 2014 Revised: February 25, 2015

Accepted: March 18, 2015 Available online: September 30, 2015

$\triangle$ Correspondence: Bung-Nyun Kim, MD

Division of Child and Adolescent Psychiatry, Department of Psychiatry, Seoul National University Hospital, 101 Daehak-ro, Jongno-gu, Seoul 03080, Republic of Korea

Tel: +82-2-2072-3647, Fax: +82-2-747-5774, E-mail: kbn1@snu.ac.kr

(a) This is an Open Access article distributed under the terms of the Creative Commons Attribution Non-Commercial License (http://creativecommons.org/licenses/by-nc/3.0) which permits unrestricted non-commercial use, distribution, and reproduction in any medium, provided the original work is properly cited. est in $\mathrm{Val} / \mathrm{Val}$ homozygotes and lowest in Met/Met homozygotes. ${ }^{5}$ Due to its important role in the prefrontal dopamine and norepinephrine neurotransmission, COMT is considered as an essential candidate gene for the etiology of ADHD.

To date, studies on the association between the COMT $\mathrm{Val}^{158}$-Met polymorphism and ADHD reported conflicting findings. ${ }^{6}$ Various races/ethnicities of the analysis population and different designs for genetic associations across studies have potential for conflicting results. For example, the COMT $\mathrm{Val}^{158}$-Met polymorphism was found significantly associated with $\mathrm{ADHD}$, with the over-transmission of Met allele in a family-based association study carried out in 166 German children. ${ }^{7}$ In contrast, other family-based association study carried out in 48 Jewish children claimed an association in completely the opposite direction (over-transmission of Val allele in ADHD probands). ${ }^{8}$ In a Korean study, there was no difference in COMT allele frequencies between 60 patient group recruited from Cheonan City and Jeonju and 100 control group recruited from Pyungtaek City, although there was a significant difference of COMT genotype frequencies (lower frequencies of Met/Met genotype and Val/Val genotype in 
Table 1. Allelic transmission calculated by extended transmission disequilibrium test for COMT Va/ ${ }^{158}$-Met polymorphism (number of trios=181)

\begin{tabular}{lccccccc}
\hline Markers & Minor allele & Major allele & T:NT & $\chi^{2} / \mathrm{df}=1$ & $\mathrm{p}$ & $\mathrm{RR}$ \\
\hline rs1191454 & Met & Val & $73: 100$ & 4.21 & 0.040 & 0.73 \\
\hline
\end{tabular}

NT: not-transmitted minor allele count, RR: relative risk, T: transmitted minor allele count

ADHD children). ${ }^{9}$ This study is limited by small sample size of ADHD population and non-matching for geographic region between the patient and control populations that might cause the problem of population stratification..$^{10,11}$

The transmission disequilibrium test (TDT) is a commonly used method for controlling for confounding due to unaccounted population admixture in genetic association studies. ${ }^{11,12}$ TDT avoids the problem of population stratification by using un-transmitted alleles as internal controls for transmitted alleles from heterozygous parents to affected offspring. Therefore, in this study, we investigated the association between the COMT Val ${ }^{158}$-Met genotype and ADHD through the TDT, in a larger Korean population than the previous study. ${ }^{9}$

\section{METHODS}

\section{Subjects}

Subjects with ADHD and both biological parents were recruited from child psychiatric clinics in Korea. In order to confirm ADHD diagnoses and any comorbid disorders, all subjects were administered the Korean Kiddie-Schedule for Affective Disorders and Schizophrenia-Present and Lifetime Version. ${ }^{13}$ Exclusion criteria included the following: 1) a history of pervasive developmental disorder, mental retardation, bipolar disorder, psychotic disorder, obsessive compulsive disorder, or Tourette's syndrome; 2) a history of neurological disorder; 3) an intelligence quotient (IQ) below 70; and 4) the presence of learning disabilities or language disorders. We provided detailed information about the study to the parents and children and then obtained written informed consent before any child entered the study. The study protocol was approved by the institutional review board of the Seoul National University Hospital.

\section{COMT genotyping}

\section{Genomic DNA was extracted from blood (stored frozen) using a G-DEX}

TM II Genomic DNA Extraction Kit (Intron, Korea) according to the manufacturer's protocol. SNP detection was based on an analysis of primer extension products generated from previously amplified genomic DNA using a chip-based MALDI-TOF mass spectrometry platform (Sequenom, Inc., CA, USA). The general procedures were performed according to the manufacturer's standard protocol. The oligonucleotide primers (5'-ACG TTG GAT GTT TTC CAG GTC TGA CAA CGG and 5'-ACG TTG GAT GAC CCA GCG GAT GGT GGA TTT) were used to generate polymerase chain reaction (PCR) products.

\section{Statistical analysis}

A family-based study was performed to assess the genetic association using transmission disequilibrium test (TDT) statistics. The '--perm' option was used to generate a single-point empirical $p$ value (EMP1) to correct for the non-normal trait distribution using the adaptive permutation approach. The criterion for significance was set at an EMP1 of $<0.05$. HardyWeinberg equilibrium (HWE) and Mendelian errors were examined using the PLINK options '--hwe' and '--me'. Data handling and management were performed by R packages under the LINUX environment. Given the number and the structure of our ADHD families, we used the program Genetic Power Calculator ${ }^{14}$ to calculate power at the alpha level of 0.05 .

\section{RESULTS}

A total of 181 children with ADHD (mean age $=8.92 \pm 2.61$ years, 152 boys and 29 girls) participated in this study. The combined type was the most common in our sample (59.7\%), followed by the predominantly inattentive (27.1\%) type. The ADHD, predominantly hyperactive-impulsive type and the ADHD, not otherwise specified (NOS) type were identified in $5.0 \%$ and $8.3 \%$ of the sample, respectively. The most common comorbidity was oppositional defiant disorder (14.4\%), followed by anxiety disorder (7.7\%), tic disorder (4.4\%), and depressive disorder (3.9\%), respectively.

The frequencies of Met (minor) allele and Val (major) allele were 0.301 and 0.691 for ADHD patients and 0.300 and 0.700 for their parents. The SNP passed the HWE test $(\mathrm{p} \leq 1 \mathrm{e}-06)$ and all of the family data were free from Mendelian errors. The TDT analysis of the COMT Val ${ }^{158}$-Met polymorphism showed the biased transmission of the Val allele $\left(\chi^{2}=4.21, \mathrm{p}=0.040\right)$ (Table 1). This biased transmission of the Val allele was statistically significantly associated with adaptive permutations $(\mathrm{EMP1}=0.041)$.

\section{DISCUSSION}

In this study, we found preliminary evidence of the over- 
transmission of the Val allele at the COMT Val ${ }^{158}$-Met polymorphism in Korean children with ADHD. Although the results of pooled analysis of 18 studies investigating the association between the COMT Val/Met polymorphism and ADHD did not support an association between the COMT $\mathrm{Val}^{158}$-Met polymorphism and $\mathrm{ADHD}$ (pooled $\mathrm{OR}=1.02,95 \%$ CI $0.92-1.13, \mathrm{p}=0.727$ ), biochemical, animal, pharmacologi$\mathrm{cal}$, and neuroimaging studies indicate the involvement of the COMT gene in ADHD. ${ }^{6}$ Over-transmission of the high enzyme activity allele (Val) in children with ADHD is plausible because of its involvement in catecholamine metabolism and prefrontal dopamine-lowering effect. ${ }^{4,5}$

Inconsistent with a previous Korean study that had reported no relationship between ADHD and the COMT gene, ${ }^{9}$ we found the over-transmission of Val allele in $\mathrm{ADHD}$ patients. Previous study used case-control association analyses and we used family-based association analyses. In the analysis of 93 investigations where both unrelated case-control and familybased design had been employed, Evangelou et al. ${ }^{15}$ concluded that unrelated case-control and family-based designs give overall similar estimates of association. Although populationbased association studies are generally regarded as more statistically powerful than family-based studies, ${ }^{16}$ the much smaller sample size in previous study (60 ADHD patients) may mask a real association. Assuming the prevalence of 0.05 , a Val allele frequency of $70 \%$, and according to Eisenberg et al., ${ }^{8}$ a genotypic relative risk of 0.97 and 1.86 for the Val/Metgenotype and the Val/Val genotype, respectively, the 181 families used in this study have a power of $80 \%$ to detect an association at the alpha level of 0.05 . In contrast, the 60 cases and 100 controls used in previous study have a power of $47 \%$ to detect an association at the alpha level of 0.05 . In addition, there is the possibility that population stratification, one problem with the case-control design, ${ }^{10,11}$ affected the negative results of previous study. However, we cannot completely rule out the possibility that inconsistent results between previous study and our study may be due to type I error of this familybased association study.

In conclusion, the results of this study suggest that the COMT Val ${ }^{158}$-Met polymorphism is associated with ADHD in the family-based association study among the Korean ADHD population. However, we genotyped only one specific SNP of COMT, and this may have been insufficient to fully address the association between this gene and ADHD. The study must be replicated in a case-control association study and with using more SNPs.

\section{Acknowledgments}

This study was supported by a grant from the Korean Health Technology R\&D Project, Ministry of Health \& Welfare, Republic of Korea (A120013).

\section{REFERENCES}

1. Biederman J, Faraone SV. Attention-deficit hyperactivity disorder. Lancet 2005;366:237-248.

2. Faraone SV, Biederman J. Neurobiology of attention-deficit hyperactivity disorder. Biol Psychiatry 1998;44:951-958.

3. Del Campo N, Chamberlain SR, Sahakian BJ, Robbins TW. The roles of dopamine and noradrenaline in the pathophysiology and treatment of attention-deficit/hyperactivity disorder. Biol Psychiatry 2011;69:e145e157.

4. Napolitano A, Cesura AM, Da Prada M. The role of monoamine oxidase and catechol O-methyltransferase in dopaminergic neurotransmission. J Neural Transm Suppl 1995;45:35-45.

5. Lachman HM, Papolos DF, Saito T, Yu YM, Szumlanski CL, Weinshilboum RM. Human catechol-O-methyltransferase pharmacogenetics: description of a functional polymorphism and its potential application to neuropsychiatric disorders. Pharmacogenetics 1996;6:243-250.

6. Sun H, Yuan F, Shen X, Xiong G, Wu J. Role of COMT in ADHD: a systematic meta-analysis. Mol Neurobiol 2014;49:251-261.

7. Palmason H, Moser D, Sigmund J, Vogler C, Hanig S, Schneider A, et al. Attention-deficit/hyperactivity disorder phenotype is influenced by a functional catechol-O-methyltransferase variant. J Neural Transm 2010; 117:259-267.

8. Eisenberg J, Mei-Tal G, Steinberg A, Tartakovsky E, Zohar A, Gritsenko I, et al. Haplotype relative risk study of Catechol-O-Methyltransferease (COMT) and attention deficit hyperactivity disorder (ADHD): association of the high-enzyme activity Val allele with ADHD impulsive-hyperactive phenotype. Am J Med Genet 1999;88:497-502.

9. Song EY, Paik KC, Kim HW, Lim MH. Association between CatecholO-Methyltransferase gene polymorphism and attention-deficit hyperactivity disorder in Korean population. Genet Test Mol Biomarkers 2009;13:233-236.

10. Cardon LR, Palmer LJ. Population stratification and spurious allelic association. Lancet 2003;361:598-604.

11. Hattersley AT, McCarthy MI. What makes a good genetic association study? Lancet 2005;366:1315-1323.

12. Wang WY, Barratt BJ, Clayton DG, Todd JA. Genome-wide association studies: Theoretical and practical concerns. Nat Rev Genet 2005;6:109118.

13. Kim YS, Cheon KA, Kim BN, Chang SA, Yoo HJ, Kim JW, et al. The reliability and validity of Kiddie-Schedule for Affective Disorders and Schizophrenia-Present and Lifetime Version- Korean version (K-SADSPL-K). Yonsei Med J 2004;45:81-89.

14. Purcell S, Cherny SS, Sham PC. Genetic Power Calculator: design of linkage and association genetic mapping studies of complex traits. Bioinformatics 2003;19:149-150.

15. Evangelou E, Trikalinos TA, Salanti G, Loannidis JP. Family-based versus unrelated case-control design for genetic associations. PloS Genet 2006;2:e123.

16. Risch NJ. Searching for genetic determinants in the new millennium. Nature 2000;405:847-856. 